\title{
BULK RELATIVISTIC MOTION IN A COMPLETE SAMPLE OF FLAT SPECTRUM RADIO SOURCES
}

C.J. Schalinski, A. Witzel, Th.P. Krichbaum, C.A. Hummel, P.L. Biermann Max-Planck-Institut für Radioastronomie, Bonn, F.R.G.

K.J. Johnston, R.S. Simon

E.O. Hulburt Center for Space Research, N.R.L., Washington, D.C., U.S.A.

As part of a multi-epoch and multi-wavelength study of the physics of core dominated radio sources we have investigated the occurrence of apparent superluminal motion and other indications for bulk relativistic motion (b.r.m) in a statistically complete, flux density limited $\left(S_{5 G H z} \geq 1 J y\right)$ sample of 13 objects with flat radio spectra $\left(\alpha_{5 G H z}^{2.7 G H z} \geq-0.5, S_{\nu} \sim \nu^{\alpha}\right)$, $\delta \geq 70^{\circ}$ and $\left|b_{I I}\right| \geq 10^{\circ}$. These sources come from the S5-survey (Kühr et al., 1981) and are optically identified as 7 quasars and 6 BL-Lac objects. They have been observed over a wide range of frequencies, from radio through X-rays (s. Eckart et al.,1986 and references therein). Radiomaps have been obtained at frequencies from $327 \mathrm{MHz}$ to $22.2 \mathrm{GHz}$ with resolutions from arcseconds to 0.2 mas, using the VLA, MERLIN and telescopes of the US- and European-VLBI networks. A recent status report on the VLBI-observations is given by Witzel (1987). In this paper we summarize the results on the direct evidence for b.r.m. in this sample as derived from repeated VLBI-observations at $5 \mathrm{GHz}$, as well as supporting evidence from SSC-calculations and flux density variability of the $5 \mathrm{GHz}$ VLBI core components (Table 1).

For the sources with unknown redshift we list in col.6 of Table 1 the "critical" redshifts beyond which the measured separation rate translates into $v>c\left(H_{0}=\right.$ $100 \mathrm{~km} / \mathrm{s} / M p c, q_{0}=0.5$ ). Deep optical imaging (Kühr, priv. com.) gives evidence for

\begin{tabular}{llccccccc}
\multicolumn{7}{c}{ Table 1 : } \\
\hline SOURCE & $I D$ & $\begin{array}{c}N(V L B I) \\
{[5 G H z]}\end{array}$ & $\begin{array}{c}d \theta / d t \\
{[\mathrm{mas} / y r]}\end{array}$ & $z$ & $z_{\text {crit. }}$ & $\begin{array}{c}v_{\text {app }} / c \\
h^{-1}\end{array}$ & $D_{S S C}^{\min }$ & $\begin{array}{c}D_{v a r}^{\min } \\
h^{2 / 3}\end{array}$ \\
\hline $0016+73$ & $Q$ & 1 & & 1.76 & & & 8.0 & \\
$0153+74$ & $Q$ & 3 & $<0.03$ & 2.34 & & $<1.3$ & & \\
$0212+73$ & $B L$ & 3 & 0.09 & 2.37 & & 3.9 & 2.4 & 1.4 \\
$0615+82$ & $Q$ & 2 & 0.05 & 0.71 & & 1.1 & 1.3 & \\
$0836+71$ & $Q$ & 3 & 0.15 & 2.16 & & 6.2 & 4.0 & 1.9 \\
$1039+81$ & $Q$ & 2 & 0.08 & 1.26 & & 2.5 & 2.1 & 1.2 \\
$1150+81$ & $Q$ & 3 & 0.13 & 1.25 & & 4.1 & 2.2 & 1.6 \\
$1803+78$ & $B L$ & 3 & $<0.03$ & $0.68^{\natural}$ & & $<0.6$ & 4.4 & \\
$1928+73$ & $Q$ & 5 & 0.60 & 0.30 & & 7.0 & 1.7 & 1.2 \\
\hline $0454+84$ & $B L$ & 2 & 0.15 & & 0.16 & & 2.1 & \\
$0716+71$ & $B L$ & 2 & 0.09 & & 0.28 & & 1.4 & \\
$1749+70$ & $B L$ & 2 & 0.10 & $>0.7^{b}$ & 0.25 & $>2.2$ & 1.3 & \\
$2007+77$ & $B L$ & 4 & 0.30 & & 0.07 & & 2.8 & \\
\hline
\end{tabular}

redshifts from ( ( ) C.Lawrence (priv.com.),(b) Wrobel et al. (this vol.)

71

M. J. Reid and J. M. Moran (eds.), The Impact of VLBI on Astrophysics and Geophysics, 71-72.

(c) 1988 by the IAU. 
$z>0.3$ in these sources. The results of Table 1 indicate: $\{1\} 10$ out of 12 sources measured at least twice with VLB-interferometry at $5 \mathrm{GHz}$ are candidates for apparent superluminal motion - 4 out of 6 sources with known $z$ measured at least 3 times with VLBI show apparent faster than light motion -. $\{2\} 12$ out of the 13 sources show evidence for b.r.m. on the basis of SSC- calculations (s. Doppler-factors D in col.8 of Table 1). $\{3\}$ For the sources with known $z$ this is in good agreement with the Doppler-factors (col.9), derived from the flux density variability of the $5 \mathrm{GHz}$ core components adopting light travel time arguments (obviously, insufficient sampling has to be taken into account). A detailed discussion will follow (Schalinski et al.,in prep.). Thus, we conclude that b.r.m., especially apparent superluminal motion, is a common phenomenon among compact flat spectrum radio sources.

For 2 sources, $0153+74$ and $1803+78$, we derived upper limits on $v / c$. In the case of the quasar $0153+74(z=2.34)$ we detected 2 compact components $A$ and $B$ (s.Fig.1) - $A$ has an inverted, $B$ a flat spectrum between 1.6 and $5 \mathrm{GHz}$ - with a separation of about 10 mas, and a "bridge" of steep spectrum components with a bending of $\sim 180^{\circ}$. As $A$ and $B$ remained constant within the errors during almost 7 years, and neither SSCcalculations nor variability show evidence for Doppler-boosting, this source holds promise to be subluminal.

$1803+78$ (s.Fig.1: Schalinski et al.,this vol.) appears to be subluminal on the basis of observations at frequencies lower than $5 \mathrm{GHz}$, thus showing a discrepancy between expected inverse Compton- and observed X-ray flux densities. However, since the source was detected on transatlantic baselines at $43 \mathrm{GHz}$ and with IRAS (s.Witzel,1987 for refs.), and recent $22.2 \mathrm{GHz}$-VLBI observations indicate the presence of a new component close to the core, relativistic effects cannot be excluded and require further studies at higher frequencies.

\section{REFERENCES}

Eckart A.,Witzel A.,Biermann P.,Johnston K.J.,Simon R.,Schalinski C.J.,

Kühr H., 1986 : Astron. \& Astrophys., 168,17

Kühr H.,Pauliny-Toth I.I.K.,Witzel A.,Schmidt J., 1981: A.J., 86, 854

Witzel A., 1987: in Superluminal Radio Sources, ed. J.A. Zensus and T.J.

Pearson (Cambridge: Cambridge University Press),p. 83
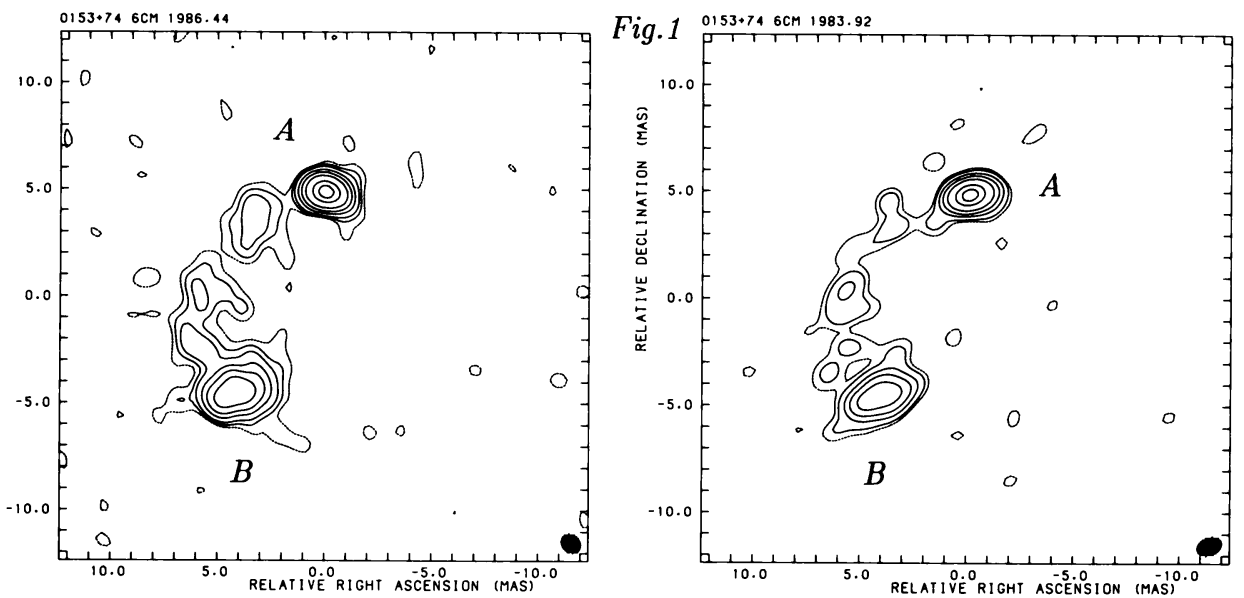